\begin{tabular}{ccc}
\hline \multicolumn{3}{c}{ ANNALES } \\
UNIVERSITATIS MARIAE CURIE-SKŁODOWSKA \\
LUBLIN - POLONIA \\
VOL. LXVIII, 1-2 & SECTIO AA & 2013 \\
\hline
\end{tabular}

\title{
Polymer dimensional changes in optical cables
}

\author{
Mateusz Lutomski ${ }^{\mathrm{a}}$ and Barbara Gawdzik ${ }^{\mathrm{b}}$ \\ Department of Polymer Chemistry, Faculty of Chemistry, \\ Maria Cure-Sklodowska University \\ Gliniana 33, 20-614 Lublin, Poland, \\ ${ }^{a}$ Mateusz.lutomski@corning.com, \\ barbara.gawdzik@poczta.umcs.lublin.pl
}

\begin{abstract}
Optical cables and fibers are extremely sensitive for mechanical, thermal and environmental conditions, which can affect their optical performance. This article describes known reasons and mechanisms responsible for dimensional changes in temperatures cycling, which can influence optical and mechanical performance and properties of the cables, including internal cable components. Understanding all physical and chemical mechanisms which meet different manufacturing parameters, various materials properties and external conditions allows to control and reduction of cable shrinking and as a result, improving mechanical and optical performance of optical cables.
\end{abstract}

\section{INTRODUCTION}

All polymers shrink. Depending on the purpose, such phenomena may be an advantage (heat shrinks, etc.), but usually, such effect is unwanted and can cause many problems and failures in various industrial fields. Production of Fiber Optical cables, which are very sensitive to mechanical stresses, forces better understanding of such phenomena. Especially as when the market grows and higher transmission is needed, smaller dimensions of cables and lower prices are required. But still, all cables need to fulfill international standard requirements. Optical 
performance is precisely defined, so special mechanical performance should be provided. Indoor cables should retain flame retardant behavior, outdoor, be proofed for environmental and chemical conditions. Also, they should be environmentally friendly and meet RoHS (RoHS stands for Restriction of Hazardous Substances) and REACH (Registration, Evaluation, Authorization, Restriction of Chemicals) requirements. Finally, materials for cable jacket cannot be very expensive but need to perform very steadily during production... and don't shrink too much.

After the rapid technical evolution in the early 80's, attenuation performance was dramatically improved, and the cost of the fiber was significantly reduced. However, shrinking can affect those improvements, causing many issues with cable and fiber performance. Shrinking jacket can increase attenuation. It can also expose ends of fibers (which practically don't shrink) to external humidity and other rough conditions. Different materials, and consequently cable elements, can shrink in a different way. The problem also affects loose tubes and tight buffers, connectors, fanouts and other cable elements. But what is shrinking, shrinkage, shrinkback, and why does it happen? This review will collect information regarding the shrinking phenomena, the nomenclature from optical cables' industry perspective, the reasons which can cause different shrinking, and known methods of controlling and reducing this unwanted behavior on various materials and components. As market started to grow, cable manufacturers increased emphasis on costing and performance optimization. More innovations on polyolefin market was made. Special kind of MDPE (Medium Density Polyethylene) was developed [1], especially for outdoor optical cables, with low temperature modulus properties and a low post extrusion shrinkage, characteristic to minimize the axial compressive stress exerted by the jacketing on the cable core. Also, black version of HDPEs (High Density Polyethylene) [2-6] was used with low compressive stresses for lowest post extrusion shrinkage. Nowadays, most conventional communication cables for outdoor use are made of polyethylene (PE) provided with UV-resistant additive like black carbon. For indoor cables, different kind of materials are used. Flame retardant materials are much more complex, and their shrinking behavior is harder to predict. Also, depending on their composition, production date or even storage conditions, same materials can act differently during manufacturing process. Cables for indoor use had Flame Retardant, PVC (polyvinyl chloride) jackets. PE and PVC materials for jackets of communication cables have now been standardized internationally, (the main standard in Europe is/was EN 
50290-2-27). Polyethylene is a very good sheathing material for outdoor use, but it is not flame-retardant. In other words, it does contribute to the spread of fire. In US, PVC is physically an appropriate jacketing material with a high degree of flame-retardancy, but in the event of fire, it generates black smoke, and poisonous and corrosive gas. Such emissions are harmful not only to electrical components, but can also cause death and are prohibited in some European countries. Due to the smoke emission and toxic gas set free when PVC burns, halogen-free (FRNC/LSNH) sheathing compounds have become more and more specified and available (The definition of FRNC/LSNH is FlameRetardant, Non Corrosive, Low Smoke, No Halogen).

FRNC materials usually consist of base polymer like EVA (Ethylene Vinyl Acetate), elastomers, flame retardants $\left(\mathrm{Al}(\mathrm{OH})_{3}, \mathrm{ATH}, \mathrm{Mg}(\mathrm{OH})_{2}\right.$, huntite and hydromagnesite, various hydrates, boron compounds and much more). Such complex composition of organic and inorganic compounds affects as well cables performance and shrinking behavior. Another example of cable components which shrinking can reduce cable performance are buffer tubes. Although the buffer tube is within a cable, the shrinkage is limited by the structure of the cable it still can cause some problems during connecting cable to closures or data centers. However the problem might appear just after tube production, or even during manufacturing. Different materials, construction types and tube diameters, requires special conditions, with very narrow temperature ranges and strictly defined production parameters.

Various cable components can shrink in various ways, because of different polymers structure and properties. All plastics parts shrink after processing, as a result of their compressibility and the thermal contraction as they cool from the processing temperature. Because crystallites contain more ordered and better packing of the polymer chains, phase transition increases shrinkage significantly. For amorphous polymers, shrinkage values are not only low, but shrinkage itself is quick to occur. Crystalline polymers are not only affected by compressibility and temperature shrinkage, but also by crystallization shrinkage. As the polymer solidifies, crystals form and the improved packing leads to shrinkage values far greater than those seen in amorphous polymers. For example, typical crystalline polymer such as PP (Polypropylene), shrinkage can be between 5 -10 times the shrinkage of an amorphous polymer [7]. Only about $85 \%$ of this higher shrinkage will have taken place in the first 24 hours, about 98$99 \%$ will have taken place in the first week and the remaining shrinkage may take up to 3 months to complete. In such cases, annealing for a short 
time at the maximum crystallization temperature to force the polymer to equilibrium where full shrinkage can take place within an hour.

\section{SHRINKING}

Shrinking behavior has been widely described in literature for many years [1-13]. Depending on manufacturing field, country or even company, phenomena is named and understood differently. That is not surprising when such data is compared. Shrinking consists of many mechanisms and appears in different conditions, even when two extrusion lines seems to be copied at the same way. It can change the molecular structure, can be reversible and irreversible, can be partially effect of production parameters, material individual behavior (polymer chains orientation and crystallinity level), temperature, time, external conditions, production line construction and speed, equipment type (and life time) or even individual experience of manufacturing engineer. Because of such complexity of a problem special nomenclature based on world literature will be implemented to easier understanding and distinguish those specific effects.

\section{THERMAL EXPANSION AND CONTRACTION}

Thermal expansion is the tendency of matter to change in volume in response to a change in temperature [8].

When a substance is heated, its particles begin moving more than usual, maintaining a greater average separation. The degree of expansion divided by the change in temperature is called the material's coefficient of thermal expansion and generally varies with temperature. When heat is added to most materials, the average amplitude of the atoms vibrating within the material increases. Such effect increases the separation between the atoms causing the material to expand. If the material does not go through a phase change, the expansion can be easily related to the temperature change. The linear coefficient of thermal expansion $(\alpha)$ describes the relative change in length of a material per degree temperature change. a is the ratio of change in length $(\Delta l)$ to the total starting length $\left(l_{i}\right)$ and change in temperature $(\Delta T)$.

$$
\alpha=\Delta l / l_{i} \cdot \Delta l
$$

The linear coefficient of thermal expansion [7]. 
If the linear coefficient of thermal expansion is known, the change in the sample length can be theoretically calculated for each degree of temperature change. This effect also works in reversal. If energy is removed from a material, then the object's temperature will decrease, causing the object to contract. Coefficient of thermal expansion can serve as a material characteristic only where material behavior is reversible. But in world of polymers other mechanisms appears parallel which complicates the topic much more.

\section{SHRINKAGE AND SHRINKBACK}

The most accurately described effect of shrinking, called shrinkage, consist of a couple other effects, which in combination creates shrinkage. Shrinkage of the cable jacket, commonly referred to as "shrinkback", occurs as this frozen-in polymer orientation relaxes in the solid state [8]. The name shrinkback was firstly used when shrinking behavior of cable jacket was observed, revealing cable ends.

But it would be easier to understand real products' behavior, when we separate those effects theoretically. Going by that way in this review, the whole shrinking effect would be called shrinkage. Technically, shrinkage is temperature dependent volume change. When polymer is slowly cooled down to room temperature, its density changes significantly. Cooling too quickly can prevent the polymer from reaching its final density. Re-heating the cable jacket sometime later may allow plastic to continue its thickening. Two main effects which create shrinkage are shrinkback and aforementioned contraction [2]. The name "shrinkback" appears especially in cables industry publications and literature, as well as in copper transmission cables and in fiber optic cables (FOC). Shrinkback is a kind of response for frozen forces which appeared during extrusion process, while temperature increase and molecules chains have more opportunities to change configuration and come back to a shorter chain [19]. Extrusion process stretched those chains' elongating and orientating them in extrusion direction. High temperature and pressure keep them that away until the temperature is rapidly cooled down. Polymer chains do not have enough time to relax and almost immediately (depending on conditions and dimensions) are frozen in a tensed way. This tension does not disappear and is still trapped in polymer structure, waiting for an opportunity to relax. Such chance appears when cable jacket is heated up and two mechanisms met even 
though they might be contrary. Cable jacket during heating grows as a result of reversible thermal expansion until polymer chains have more movement possibilities as polymer is getting more "rubber like" and shrinkback appears. Even though sample is still expanding, opposite force appears, as stretched polymer chains are relaxing. Depending on polymer properties such effect might be correlated with glass transition effect.

If polymer is semi crystalline, during cooling the crystallization also occurs, which also affects shrinking. And at this point, it is necessary to come back to the structure of polymers. Thermoplastic polymers shrinkage also depends on the matrix morphology - amorphous or semicrystalline. In semi-crystalline polymers, the volumetric shrinkage results from the densification upon crystallization (with crystals being of higher density than the amorphous phase f.e. LDPE vs HDPE, or PP mentioned before), in addition to the shrinkage because of lower temperature. In amorphous polymers, the shrinkage is only due to the latter. The total shrinkage for semi-crystalline matrices can even be ten times higher than for amorphous.

Below $\mathrm{Tg}$ (glass transition temperature), there is virtually no molecular motion on a local scale. Polymers have many of the properties associated with ordinary organic glasses, including hardness and stiffness. The crystalline melting point is the temperature at which crystals melt, and a crystalline polymer resembles an amorphous polymer, which has no short-range order. Tm (melting temperature) generally increases as the degree of crystallinity increases. Above Tm, no crystallization exists, and below $\mathrm{Tg}$, no further movement of molecules is possible to nucleate or grow crystals. The fastest rate of crystallization occurs midway between $\mathrm{Tg}$ and $\mathrm{Tm}$. The longer a polymer remains between the two, the greater the amount of crystallization [8].

Shrinkback is a permanent effect [13], and is not reversible. The reason of this is that during relaxation, polymer changes the chain configuration. Opposite to shrinkback, contraction is irreversible and chain configuration does not change, only molecules get shorter. Both effects combined together create the shrinkage.

Depending on the temperature range and the time influence, the overall shrinkage process involves a rapid initial stage contraction of the molecular network, associated with disorientation in the amorphous phase, followed by a crystallization stage during which chain folding takes place. Crystallization can occur simultaneously with amorphous disorientation, and once the crystallization starts, further shrinkage is hindered [9]. 
Changing temperatures increase and speed up this effect. But even in room temperatures, the molecules would like to come back to reduce frozen tension. Of course, because of limited molecule movement in the solid state process is not so fast, and it needs more time to shrink. It can cause problems mentioned before in the longer time period. That is why aging is performed to predict such behavior. To understand it better, the terms from literature can be used as well, like the primary and the secondary shrink [3]. Standard DIN 16901 suggest to take the first length measurement not sooner than $16 \mathrm{~h}$ after the production. Such long time is needed for strain relaxation, and such shrink is a "primary shrink". Further uncontrolled transformation, which can be couple hundred hours long, is a secondary shrink, where recrystallization, relaxation and participles accumulation take place. As a consequence, energetic equilibration is achieved and strains are relieved. It is believed that sum of those two factors is (approximately) stable, and it is important to achieve highest primary shrink value, which could be controlled during the cooling, to obtain the smallest value of secondary shrink, which is unknown. There is another factor which significantly influences shrinkback - friction between cable elements. Such effect on one hand reduce shrinking of the jacket, but on the other also blocks relaxation in some way and helps creating frozen in stresses.

\section{RESIDUAL STRESSES}

The strains which can be blocked during polymer cooling can be also separated in respect of transition stage and physical mechanism [2, 3]. Flow Induced Residual Stress takes place, when molted polymer molecules are unstressed, and they tend to an equilibrium, random coil state. During processing, the polymer is sheared and elongated, and the molecules are oriented in the flow direction. If solidification occurs before the polymer molecules are fully relaxed to their state of equilibrium, molecular orientation is locked within the extruded part [17]. Another is the Thermal Induced Residual stress which arises during the cooling stage [2]. During the cooling stage, the polymer cools at different rates. When the polymer starts to cool, the external surface layers start to shrink, while the bulk of polymer is still hot at the core and free to contract. Later, when the internal core cools, it's contraction is constrained by the external layers since they are already rigid. 
The problem of thermal residual stresses can be especially found in the extruded polymer products where rapid inhomogeneous cooling is a part of the manufacturing process. For cables, cooling starts at the outer layers of the cable jacket, where the temperature drops almost instantaneously from well above the melting point to room temperature. As a result, the outer layers stiffens, but the inner layers still occupy a larger volume, introducing stresses into the cable jacket or tube. As cooling proceeds radially, solidification produces further thermal stresses. Usually thermal stresses partially persist in the finished product since most polymers are viscoelastic. Furthermore, the stress development is also influenced by the semi-crystalline microstructure of certain polymers like PE. Moreover, high (or "too high") temperature of jacketing process can have an impact on inside cable components like buffer tubes and other materials which could be sensitive for such temperature treatment.

\section{ORIENTATION AND CRYSTALLIZATION}

Orientation is frozen-in stretching or elongation of the polymer molecules occurring during polymer flowing from the extrusion die to windup area of the extrusion process. On removal of the deforming stresses, the molecules start to coil up again, but the process may not go to equilibrium before the polymer cools to below its glass transition temperature ( $\mathrm{Tg}$ ). This leads to residual orientation (frozen-in strain) and aforementioned corresponding frozen-in stresses. Usually polymers with extensive side branches or bulky side branches along the polymer chain can be more sensible to frozen-in elongation than polymers with smaller side chains. Same result occurs when rubbery and elastic polymers are compared with stiffer ones, which are more susceptible to frozen-in orientation.

Many different factors may affect orientation during the extrusion process [20]. When the extrudate exits the die, the polymer molecules, oriented in the die land area, relax and reentangle, causing die swell. If the extrudate is allowed to droll out the die, the cross section swells, becoming larger than the die opening due to polymer relaxation. Extrudate pulled away from the extruder, orients the polymer molecular chains in the machine direction. The draw depends on the puller speed relative to the extruder output. Draw ratio is directly related to molecular orientation, resulting in higher tensile and flexural properties in the machine direction compared to the transverse position. Process settings 
and die should be chosen very carefully. The draw ratio depends on the product size exiting the die versus the required product size [20]. On PBT material example, it has been shown that thermal shrinkage of the tubes with low orientation can be attributed to the rubber-like behavior of a molecular network [9]. The explanation of such phenomena might be that disorientation of the oriented amorphous phase is the predominant mechanism by which shrinkage occurs. Relatively slow crystallization because of low orientation, does not compete with shrinkage. In the highly drawn samples with high orientation, however, crystallization can be extremely rapid, especially at high temperatures, and can impede shrinkage. As mentioned before, shrinkback around glass temperature may be additional force which will oppose thermal expansion during first heating of the tube depending on polymer chains orientation.

\section{But how to measure orientation?}

There are many tests which have been devised [20], depending on equipment type, kind of sample material etc. One of the simplest method is called "Chrysler Test" and has a couple of variations. One of them uses measured stripes cut from a sheet (in different directions to determine local orientation!). Then the samples go to the oven at temperature of polymer thermoforming and stripes are measured again. The greater the difference in specified direction, the bigger the orientation in the sample. The other way for transparent polymers is just to observe by passing the sheet between polarized film. The orientation will appear as rainbow patterns across the sheet. One of the more complicated method and much more precise is polarized Raman Spectroscopy [15], which probes information about molecular orientation and symmetry of the bond vibrations, in addition to the general chemical identification which standard Raman provides. Measurements are made acquiring spectra with polarisation which is either parallel or perpendicular to the inherent polarisation of the excitation laser. Polarisor is inserted in the beam path between the sample and the spectrometer and Raman polarisation is selected by the user [15]. Polarization measurements provide information about molecular shape and the orientation of molecules in ordered materials, such as crystals, polymers and liquid crystals. Other methods which can be also used include Wide Angle X-Ray Diffraction, Linear Dichroism, Sonic Techniques, or Polarised Fluorescence and a described in literature $[15,20]$. 


\section{CRYSTALLIZATION}

As it was shortly mentioned, when crystalline polymers cool, small areas of short-range order form. These are highly organized and closely packed molecules which can create crystals. There are several models and theories regarding crystal formation, and the most popular is the "fringed micelle" model. It is a two-dimensional representation of molecules in a crystalline polymer according to the fringed micelle theory [16, 18]. It shows the ordered regions or crystallites embedded in an amorphous matrix. A single polymer chain may be present in sections of different crystallites, which can complicate shrinking effects even more. Larger structures, made up of bundles of crystallites, are named "spherulites" and these are formed in the bulk of the material. They behave in a similar manner to the formation and growth of grains in a metal. Those micelles can be observed on Figure 1.

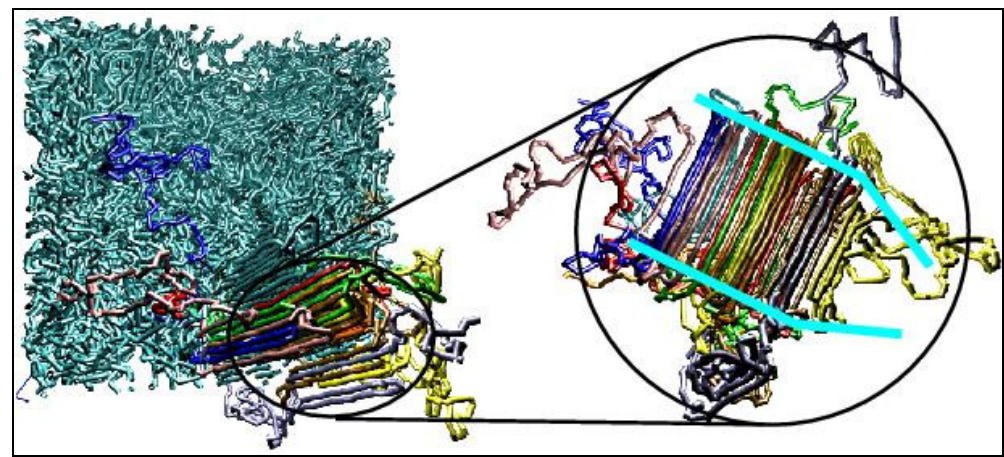

Fig. 1. Formation of a crystal lamella as observed with molecular dynamics. The crystallization domain is observed to extend over several chain diameters [12].

Crystal formation begins at the nucleation points and extends outwards into the bulk of the polymer. The nucleation can be divided into homogenous (small number nucleation sites and a few large crystals) and Heterogeneous, which consists of other elements than the primary polymer. This particles may act as nucleation sites and can form many new crystals. For example, certain coloring agents can act like that and this results in higher shrinkage rates. The type of nucleation process will also affect the properties of crystalline polymers. A product with relatively few large crystals will have different properties than one with more, but smaller crystals. 
Not all polymers crystallize to the same degree and in the same way. What is more, different measurement techniques may show different crystallinity level at the same samples. But all of polymers have "s o m e" degree of crystallinity (except for amorphous polymers of course). Crystallinity can vary between $0 \%$ (an amorphous polymer) and $80 \%$ (a highly crystalline polymer). There are also a number of variables present in polymer structure that affect crystallinity, like length of polymer chain, stereoregularity, number of polar groups and chain branching.

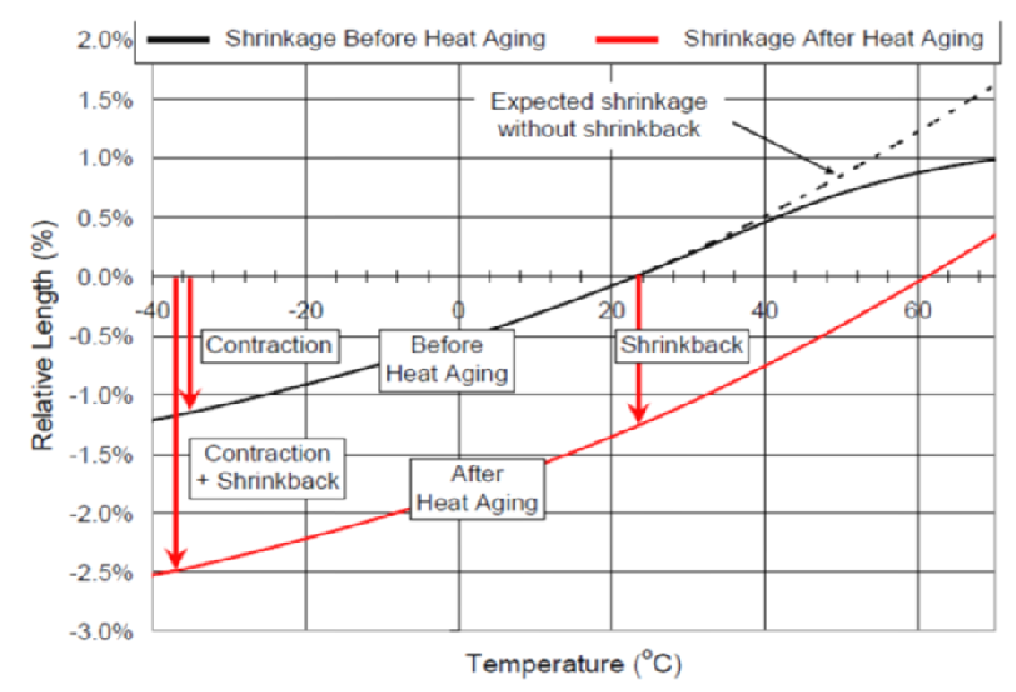

Fig. 2. Bill Hurley Corning - TMA measurement of PE cable jacket [13].

Any of these structures will behave differently in temperature cycling. Most efficient tool for such measurements is TMA (Thermo Mechanical Analysis). When measuring program is set for at least two cycles from -40 up to $50^{\circ} \mathrm{C}$ (depending on polymer type), differences between shrinkage and shrinkback can be easily observed. If production process is controlled, and various structures mentioned before could be obtained, TMA measurement of different crystallinity level and orientation could bring much more interesting conclusion. An example of TMA analysis is showed on Fig. 2.

Some TMA models with modulated measurement system allow to measure total dimensional change and its reversing and non-reversing components. Total signal is identical to standard TMA, but does not uniquely define the $T g$. Those component signals can clearly separate 
actual $\mathrm{Tg}$ from the stress relaxation event. Such type of measurement in combination with standard measurement in temperature cycle might give much more information of different shrinking effects.

\section{CONTROLLING SHRINKBACK}

By modifying processing techniques, crystallinity can be controlled, even in semi-crystalline plastics. It is possible to quench, or rapidly cool, plastic parts to reduce the formation of crystals. However, the success of this depends on the relationship of $T g$ of the polymer and the service temperature. If $T g$ is higher than the service temperature, then the quenching will prevent the formation of crystals during cooling. As a result, they are unlikely to form during the service life of the product. Equally, if the service temperature is approximately the same as or higher than $T g$, then quenching will only delay the inevitable, and crystallization and shrinkage will eventually occur afterwards, which can cause serious problems after some time [20].

Equally, it is possible to anneal polymers after processing, to ensure that proper crystallization has taken place. Annealing involves holding the polymer above $\mathrm{Tg}$ but below $\mathrm{Tm}$ for a specific time to both encourage and control the growth of the crystalline structure. High nucleation and growth rates can also be achieved if heterogeneous nucleation is used. In this case, nucleation is initiated by seeding with a foreign particle, which is typically a polymer similar to the base polymer but with a higher melting point. Some commercial products incorporate special nucleating agents to produce a high degree of crystallization and controlled structures, including coloring agents. It is also possible to induce directional crystallization by stretching polymers below $\mathrm{Tm}$ to create crystalline filaments, fibers or sheets with a crystalline structure oriented in the direction of stretching. This cold-drawing technique is used extensively in fiber and film production and in the production of PET bottles.

Another way to control shrinkage is good process controls and understanding of the extrusion process [20]. Many variables have to be controlled and taken into consideration. Even very small changes in the process may change parameters of the product, and affect the behavior of melt material, stress inside and finally, the shrinkage.

Beginning from temperature zone control, the thermocouple must operate properly. Temperature controllers have evolved over the years 
with improvements in electronics to provide very accurate control on all extruder zones.

If the actual and set temperatures in a particular zone are significantly different, it may mean i.e., that the temperature is not correctly controlled in that particular zone, or the thermocouple is not operating properly, and may need replacement. Other reason might be that the temperature setting for the material could be wrong or the excess shear heat is being generated in that zone. Also, the polymer melt temperature control is critical to the control and reproducibility of the extrusion process. Melt temperature measurement inside the extruder barrel is not always practical, because the turning screw would shear off a melt probe sticking down in the melt stream.

Melt pressure measurements at the extruder head are also very important. The melt temperature and pressure at the die produce consistent output, resulting in uniform product cross sectional dimension. Like the melt temperature, the melt pressure may show if there are any problems in the extruder and die. The Die pressure fluctuations correspond with the output fluctuations and dimensional changes. Even very small differences in the die shape may result in a bigger shrinking behavior because of the energy dissipation at the probe due to polymer shear heating resulting from the polymer flowing. Also, the usage of a die and shear rate may influence shrinkage.

Another important factor that should be investigated and controlled is rheological behavior [17]. Uncontrolled changes in polymer viscosity may result in product dimensional changes, higher or lower shear heating causing possible res in degradation, or higher motor loads and different melting and metering characteristics. Such behavior may have great importance, especially with flame retardant materials. Other than the equipment problems, viscosity variations are an issue associated with running different regrinds at various levels, flame retardant materials, blends, or coextrusions. Measurement of MFI, or online viscosity measurements could avoid or reduce such problem and detect any sudden viscosity changes. Another, but not so popular method could be online FT-IR measurements which could show any composition and additive levels in a formulation. In-line measurements eliminate the problems associated with sampling methodology, sample preparation, and time delays. In addition, the infrared (IR) crystalline absorption bands are not present, as the polymer is molten and amorphous. In-line systems must be able to handle high temperatures and high melt viscosities, and be able to perform in a manufacturing environment with delicate equipment. 


\section{SUMMARY}

Because of complexity of this phenomena, dimensional change of polymers, is still not $100 \%$ understood. The polymers might have the properties of both liquids and solids depending on their properties and conditions. All kind of described mechanisms affect dimensional changing properties of polymers. When cable production factors are added, which additionally affects orientation of polymer chains and sensitivity of optical fibers inside, preparing perfect conditions which prediction of all the consequences in short and long term, might be a real challenge. Especially after realizing how much different kind of polymer materials and components optical cables are made of. Finally - all kind of external conditions, where cables are placed also can be very harmful and can influence shrinking and as a consequence - reduce optical performance. Laboratory tests which can simulate real conditions including thermal cycling, environmental and mechanical influence should not be the only tool for such complex problem. Much more effort should be placed on experiments in real environment, including manufacturing and mounted products in long term behavior.

\section{REFERENCES}

[1] H. De Boer and O. Grosskurth, TKF BV. 7480 AA, Haaksbergen Netherlands; Ann Watson \& James Robinson Borealis Polymers NV. B-3583, Beringen Belgium "Low Shrink HDPE for the Sheathing of Fibre Optic Minicable" .

[2] L. Olasz, Doctoral thesis no. 63 "Residual Stresses and Strains in Cross-linked Polyethylene Power Cable Insulation", TRITA, HFL0412 KTH Engineering Sciences Stockholm, Sweden

[3] P. P. Parlevliet, H. E. N. Bersee and Adriaan Beukers "Residual stresses in thermoplastic composites - A study of the literature Part II: Experimental techniques" Faculty of Aerospace Engineering, Delft University of Technology Delft, The Netherlands, 4 July 2006

[4] S. T. E. Aldhouse, D. McMahon and J. E. Robinson, "Problems and Opportunities in the Use of Short Relaxation. Linear Polymers in Telecommunication Cable Applications"., Proceedings of Fourth International Conference; Plastics in Telecommunications, 1986, pp. 32.1-32.12. 
[5] L. Rogestedt and H. B. Martinsson, "A New Generation Polyethylene Resins For Cable Jacketing Applications", Proceedings of the $47^{\text {th }}$ IWCS, 1999, pp. 126-131.

[6] T. Chen and J. R. Leech, "Design of Polyethylene Cable Jacket Compounds of Superior Jacketing Performance"., Proceedings of the 48th IWCS, 1999, pp. 807-814.

[7] "Crystallinity in Plastics", Zeus Technical Newsletter, Copyright C2007-2010 Zeus Industrial Products, Inc.

[8] P. P. Parlevliet, H. E. N. Bersee and A. Beukers. "Residual stresses in thermoplastic composites - A study of the literature - Part I: Formation of residual stresses" Faculty of Aerospace Engineering, Delft University of Technology, The Netherlands. 28 December 2005.

[9] V. B. Gupta, J. Radhakrishnan and S. K. Sett, "Interaction between thermal shrinkage and crystallization in axially oriented poly(ethylene terephthalate) fibres and films", Textile Technology Department, Indian Institute of Technology, New Delhi, India 20 January 1993.

[10] M. Trznadel, T. Pakula and M. Kryszewski, Centre of Molecular and Macromolecular Studies, Polish Academy of Science, Łódź, ul. Boczna 5, Poland, 13 February 1984.

[11] M. Trznadel "Naprężenia skurczu termicznego zorientowanych polimerów" PhD thesis Polska Akademia Nauk Łódż 1989.

[12] Polymer crystallization, Équipe "Théorie et Simulation des Polymères" (ETSP),Institut Charles Sadron (ICS), Strasbourg, France.

[13] W. C. Hurley, R. S. Freeland, and M. R. Ellwanger "ShrinkbackInduced Attenuation in Loose Tube" Cables Corning Cable Systems, Hickory, NC.

[14] "Halogen-free Optical Fibre Cables for Outdoor and/or Indoor Use", November 2010, Belden application note.

[15] "What is polarized Raman spectroscopy?" Horriba Scientific Paper and webinar.

[16] S. H. Wasserman and J. LaMonte Adams, Rheology and crystallization in fiber optic cable jacket and conduit extrusion, Union Carbide Corporation, Somerset, NJ 
[17] K. Migler, Ch. Liu, and D. J. Pine, "Structure Evolution of a Polymer Solution at High Shear Rates" Exxon Research and Engineering Company, 79 Route 22 East, Annandale, New Jersey 08801Received July 13, 1995; Revised Manuscript Received November 27, 1995X

[18] G. D. Brown, K. P. (Peter) Pang, Ph.D., S. H. Wasserman, Ph.D. "Design of New Easy Processing High Modulus Compounds for Fiber Optic Cable Use".

[19] J. J. Henry, Nafaa "Low Shrinkage in Wire and Cable Extrusion and the Importance of Grade Selection" Mekhilef Arkema Inc. King of Prussia, PA 19406.

[20] H. F. Giles, Jr., J. R.Wagner, Jr. and E. M. Mount, III, "Extrusion: The Definitive Processing Guide and Handbook" 2005.

\section{CURRICULA VITAE}

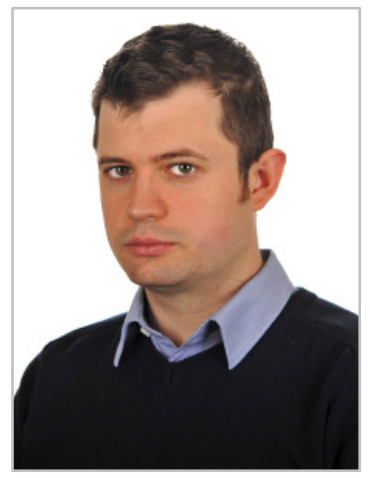

Mateusz Lutomski was born in 1983 in Lublin. After receiving M.Sc. from Maria CurieSklodowska (MCS) University in 2007 (Optical Fibers Department) he was hired as a Technical Assistant at Technical University of Lublin. At the end on 2007 he moved to Łódź where he started working for Corning Cable Systems firstly in Research and Development Department, where he prepared his first patent. In the beginning of 2010 he participated in creating Testing and Qualification Laboratory and has been responsible for Materials Laboratory.

Parallel he finished post-graduation studies at Technical University of Łódź - "Innovative materials technologies and measurementcomputational systems" (2010) and "Chemical analysis in quality controlling and environmental protection"(2011). He was promoted to Senior Materials Development Engineer in 2012 and joined Global Materials Group. He started $\mathrm{PhD}$ studies at MCS University with collaboration with Corning Cable Systems (PL \& DE) and Corning Incorporated (US) in 2012, with research area focused on "shrinkback" phenomena and dimensional polymer changes in Optical Cables. 


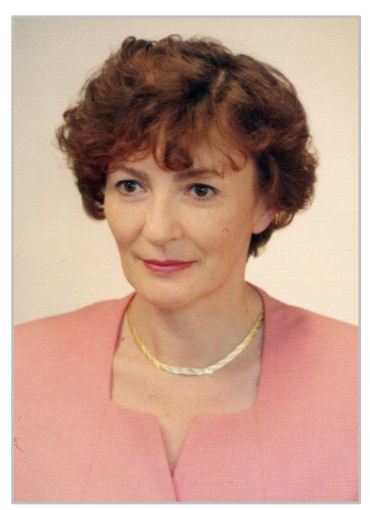

Barbara Gawdzik was born in 1954 in Lublin. After receiving M.Sc. from Maria Curie-Sklodowska (MCS) University in 1978 she was employed at the Department of Polymer Chemistry and Technology in MCS University. She received Ph.D. in 1986 and habilitation in 1993. In 2004 she was appointed by the President of Poland to the post of professor. Since 2006 has also been head of the Department of Polymer Chemistry, MCS University.

She is the author and co-author of 150 original papers in internatio-nal journals, 140 published as conference materials, and 21 patents.

Main areas of her scientific interests include:

- synthesis of new monomers and polymers,

- heterogeneous polymerization techniques,

- investigations of porous structure of polymeric materials,

- preparation of polymeric microspheres for chromatography,

- synthesis and applications of imprinted polymers,

- synthesis and investigations of carbon adsorbents from synthetic precursors. 